\title{
Correcting values of DNA sequence similarity for errors in sequencing
}

3 Timothy J. Hackmann*

4 Department of Animal Science, University of Florida, Gainesville, FL, 32611, USA

$6 \quad *$ For correspondence. E-mail thackmann@ufl.edu ; Tel. (352) 392-7566; Fax ( 352) 392-5595

8 Running title: Errors and sequence similarity 


\section{Abstract}

10 The similarity between two DNA sequences is one of the most important measures in

11 bioinformatics, but errors introduced during sequencing make values of similarity lower than they

12 should be. Here we develop a method to correct raw sequence similarity for sequencing errors and

13 estimate the original sequence similarity. Our method is simple and consists of a single equation

14 with terms for 1) raw sequence similarity and 2) error rates (e.g., from Phred quality scores). We

15 show the importance of this correction for $16 \mathrm{~S}$ ribosomal DNA sequences from bacterial

16 communities, where $97 \%$ similarity is a frequent threshold for clustering sequences for analysis.

17 At that threshold and typical error rate of $0.2 \%$, correcting for error increases similarity by 0.36

18 percentage points. This result shows that, if uncorrected, sequencing error would increase

19 similarity thresholds and generate false clusters for analysis. Our method could be used to adjust

20 thresholds for cluster-based analyses. Alternatively, because it requires no clustering to correct

21 sequence similarity, it could usher in a new age of analyzing ribosomal DNA sequences without

22 clustering. 


\section{Introduction}

25 The similarity between two DNA sequences is one of the most important measures in

26 bioinformatics. However, this measure is only as accurate as the sequences being compared. For

27 example, two identical sequences will no longer be identical (have $100 \%$ similarity) after

28 introducing sequencing errors. Errors pose a problem for determining the similarity of ribosomal

29 DNA sequences from microbial communities, in particular, where 1) errors occur at high rates

30 because of next generation sequencing and 2) biological variation is high and not easily

31 distinguished from errors.

Many methods exist for handling sequencing errors in ribosomal DNA sequences, but most involve clustering. During clustering, similar sequences are grouped together, and one sequence

34 (e.g., the most abundant) is presumed to be correct (representative of the cluster). This clustering is done by applying one similarity threshold (e.g., 97\%) $)^{1,2}$, two thresholds sequentially (e.g., $99 \%$ and $97 \%)^{3,4}$, or more complex algorithms ${ }^{5,6}$. With $16 \mathrm{~S}$ ribosomal DNA sequences, the nominal

37 purpose of clustering at $97 \%$ similarity is to create discrete groups (operational taxonomic units) for analysis, but this clustering also masks sequence error. known species of microbes) $)^{1-6}$ (Fig. 1A). Clustering cannot be calibrated with real communities because species are not known. Because real communities differ from mock communities (e.g., represents a serious weakness of cluster-based methods.

We present a simple method for handling errors in ribosomal and other DNA sequences. 


\section{Results}

48 We developed a method to correct the similarity of DNA sequences for sequencing errors. This method, which consists of a simple equation derived using Bayes's theorem, provides an estimate

50 of the original similarity (before introduction of errors). It requires only 1) raw sequence similarity

51 (after introduction of sequencing errors) and 2) error rates (which can be calculated from Phred

52 quality scores).

Using our method, we calculated the original sequence similarity, $P\left(S_{b}\right)$, expected at

54 different values of raw similarity, $P\left(S_{a}\right)$, and sequencing error rates (Fig. 5). At 25\% raw

55 similarity (that expected for two unrelated sequences), original and raw similarity were equal. At

$56>25 \%$ raw similarity, original similarity was higher than raw similarity, and the difference grew

57 larger as raw similarity and the error rate increased.

Raw similarity of $97 \%$ is a threshold for clustering 16 S ribosomal DNA sequences into the

59 same operational taxonomic unit (see inset of Fig. 5). At $97 \%$ raw similarity $\left[P\left(S_{a}\right)=0.97\right]$ and $1 \%$ error rate $\left(p_{x}=p_{y}=0.01\right)$, original similarity was 1.96 percentage points higher $\left[P\left(S_{b}\right)=\right.$ 0.9896]. Even at a $0.2 \%$ error rate, the original similarity was still 0.39 percentage points higher

$62\left[P\left(S_{b}\right)=0.9739\right]$. An error rate of $1 \%$ corresponds to a typical value for the Illumina MiSeq platform and reads of the V4 region of 16S ribosomal DNA (after merging paired end reads with USEARCH) ${ }^{5}$. An error rate of $0.2 \%$ corresponds to those same reads subjected to stringent 65 filtering (removal of reads with more than one expected error with USEARCH) ${ }^{5}$. In sum, original similarity was higher than raw similarity, even at low error rates, showing the importance in 67 correcting similarity for sequence error. 
70 introduced errors at a rate of $1 \%$. Following introduction of errors, the similarity of the reads

71 decreased (cf. Fig. 6A,B), as expected from Fig. 5. After applying our method for correcting

72 similarity, the mean similarity increased back to its original value (cf. Fig. 6A,C). When we

73 introduced errors at only $0.2 \%$, results were similar, but the variance in Fig. $6 \mathrm{E}$ and F was less than

74 in Fig. 6B and C. In sum, under the conditions of our simulation, our method provides an unbiased

75 estimate of the original sequence similarity over a range of error rates. The benefit of reducing

76 error was reducing variance in the estimate.

\section{Discussion}

78 We developed a method to correct the similarity of two DNA sequences for errors in sequencing.

79 The method is simple and requires only 1) raw (observed) sequence similarity and 2) error rates, 80 which can be calculated from Phred quality scores. This method is important to any analysis using

81 values of DNA sequence similarity. It is especially important for determining the similarity of

82 ribosomal DNA sequences from microbial communities, owing to frequent errors that are hard to 83 separate from biological variation.

Most methods for handling sequence errors with 16S ribosomal DNA sequences are based

85 on clustering ${ }^{1-6}$. One sequence, usually the most abundant, is presumed correct (representative of

86 the cluster). These methods can be evaluated with mock communities of bacteria, but there is no

87 way to test their accuracy with real communities.

Our method requires no clustering and no presumption about which sequences are correct.

89 Moreover, it shows that cluster-based methods are biased. With an error rate of $0.2 \%$, clustering

90 at an apparent (raw) similarity of $97 \%$ is in fact done at an actual (original) similarity of $97.39 \%$.

91 If error is not corrected, false clusters will be generated for analysis. 
While our method could be used to adjust similarity thresholds in cluster-based analyses,

93 it paves the way for cluster-free analysis. Analysis of diversity, for example, can be done using

94 phylogenetic trees [see ref. ${ }^{7,8}$, and tree construction requires only sequence similarities, not

95 clusters. By correcting DNA similarities for sequence errors, our method removes a major bias in

96 analysis of diversity. By accomplishing this without clustering, our method could usher in a new

97 age of analyzing ribosomal DNA sequences directly, with no clustering step needed.

\section{$98 \quad$ Methods}

99 Overview. We will derive an equation to correct raw sequence similarity for sequencing errors.

100 The derivation of this equation uses Bayes's theorem and follows from work determining the 101 probability of correct letters in paired-end sequence reads 5 .

103 position $k$ within the sequences, and sequences have a total of $n$ positions. Before sequencing

104 (introduction of errors), the letters are $X_{b}$ and $Y_{b}$. After sequencing (introduction of errors), some 105 letters change, and the set of letters becomes $X_{a}$ and $Y_{a}$. Let $S_{b}$ be letters in $X_{b}$ and $Y_{b}$ that are 106 similar (when compared at a given position $k$ ), and $S_{a}$ are the letters similar between $X_{a}$ and $Y_{a}$.

107 The letters that are dissimilar are $D_{a}$ and $D_{b}$. We partition $S_{a}$ as $S_{a 1}$, which originate from $S_{b}$, and 108 $S_{a 2}$, which originate from $D_{b}$.

Our goal is to calculate the original similarity, $P\left(S_{b}\right)$, or similarity before introduction of

111 calculate it from 1) the raw similarity, $P\left(S_{a}\right)$, or similarity after introduction of errors and 2) the 112 error rates $p_{x}$ and $p_{y}$ (defined below). 
113 Similarity at a given position k. To estimate similarity of $X$ and $Y$ in total, we will first estimate

114 the similarity at a given position in $X$ and $Y . P\left(S_{a[k]}\right)$ is the raw similarity at position $k$ and is

115 either 0 (dissimilar) or 1 (similar). Following Fig. 4A, we can partition it as

$$
P\left(S_{a[k]}\right)=P\left(S_{a 1[k]}\right)+P\left(S_{a 2[k]}\right)
$$

116 By Bayes's theorem

$$
P\left(S_{a 1[k]}\right)=\frac{P\left(S_{a 1[k]} \mid S_{b[k]}\right) P\left(S_{b[k]}\right)}{P\left(S_{b[k]} \mid S_{a 1[k]}\right)}
$$

117 and

$$
P\left(S_{a 2[k]}\right)=\frac{P\left(S_{a 2[k]} \mid D_{b[k]}\right) P\left(D_{b[k]}\right)}{P\left(D_{b[k]} \mid S_{a 2[k]}\right)}
$$

118 We note $P\left(S_{b[k]}\right)=1-P\left(D_{b[k]}\right)$, substitute eq. [2] and [3] into [1], and solve for $P\left(S_{b[k]}\right)$ to 119 give

$$
P\left(S_{b[k]}\right)=\frac{\left[P\left(S_{a[k]}\right) P\left(D_{b[k]} \mid S_{a 2[k]}\right)-P\left(S_{a 2[k]} \mid D_{b[k]}\right)\right] P\left(S_{b[k]} \mid S_{a 1[k]}\right)}{P\left(S_{a 1[k]} \mid S_{b[k]}\right) P\left(D_{b[k]} \mid S_{a 2[k]}\right)-P\left(S_{a 2[k]} \mid D_{b[k]}\right) P\left(S_{b[k]} \mid S_{a 1[k]}\right)}
$$

$$
P\left(S_{b[k]} \mid S_{a 1[k]}\right)=1
$$

123 and

$$
P\left(D_{b[k]} \mid S_{a 2[k]}\right)=1
$$

124 We partition $P\left(S_{a 1[k]} \mid S_{b[k]}\right)$ and $P\left(S_{a 2[k]} \mid D_{b[k]}\right)$ as

$$
P\left(S_{a 1[k]} \mid S_{b[k]}\right)=P(\alpha)_{[k]}+P(\beta)_{[k]}+P(\gamma)_{[k]}+P(\delta)_{[k]}
$$

125 and 


$$
P\left(S_{a 2[k]} \mid D_{b[k]}\right)=P(A)_{[k]}+P(B)_{[k]}+P(\Gamma)_{[k]}+P(\Delta)_{[k]}
$$

126

127

128

129

130

131

132

133

134 and

$$
P\left(S_{a 2[k]} \mid D_{b[k]}\right)=\frac{1}{3} p_{x[k]}\left(1-p_{y[k]}\right)+\frac{1}{3}\left(1-p_{x[k]}\right) p_{y[k]}+\frac{2}{9} p_{x[k]} \times p_{y[k]}
$$

with terms defined in Table 1 . For example, $P(\alpha)_{[k]}=\left(1-p_{x[k]}\right)\left(1-p_{y[k]}\right)$ is the probability that neither $X_{[k]}$ nor $Y_{[k]}$ change (no errors were introduced) after sequencing, given the letters were similar before sequencing (i.e., $X_{[k]}$ and $Y_{[k]}$ belong to $S_{b}$ ). The terms $p_{x[k]}$ and $p_{y[k]}$ are probabilities for change (sequencing error rate) for $X_{[k]}$ and $Y_{[k]}$, respectively. We assume all changes (errors) are substitutions (not insertions or deletions), giving one of three equally probable outcomes per position per sequence. Phred quality scores $(Q)$ can be used to calculate the error rates [e.g., $\left.p_{x[k]}=10^{\left(-Q_{X[k]} / 10\right)}\right]$.

Substituting expressions for Table 1 into eq. [7] and [8] gives

$$
P\left(S_{a 1[k]} \mid S_{b[k]}\right)=\left(1-p_{x[k]}\right)\left(1-p_{y[k]}\right)+\frac{1}{3} p_{x[k]} \times p_{y[k]}
$$

135 By substituting eq. [5], [6], [9], and [10] into eq. [4], we yield

$$
P\left(S_{b[k]}\right)=\frac{9 P\left(S_{a[k]}\right)-3 p_{x[k]}-3 p_{y[k]}+4 p_{x[k]} \times p_{y[k]}}{9-12 p_{x[k]}-12 p_{y[k]}+16 p_{x[k]} \times p_{y[k]}}
$$

136 The derivation of eq. [11] is equivalent if we follow Fig. $4 \mathrm{~B}$ and partition as $P\left(D_{a[k]}\right)$ as $P\left(D_{a[k]}\right)=P\left(D_{a 1[k]}\right)+P\left(D_{a 2[k]}\right)$ (not shown).

Similarity across all $\mathbf{n}$ positions. To estimate similarity of $X$ and $Y$ in total, we average $P\left(S_{b[k]}\right)$ across all $n$ positions

$$
P\left(S_{b}\right)=\sum_{k=0}^{n}\left[P\left(S_{b[k]}\right)\right] \frac{1}{n}
$$


140 This approach assumes all changes (errors) occur independently (i.e., an error occurring at $k=0$

141 does not change the probability of an error at $k=1$ ). Eq. [12] can be expanding by substituting

142 in eq. [11], giving

$$
P\left(S_{b}\right)=\sum_{k=0}^{n}\left[\frac{9 P\left(S_{a[k]}\right)-3 p_{x[k]}+4 p_{x[k]} \times p_{y[k]}-3 p_{y[k]}}{9-12 p_{x[k]}-12 p_{y[k]}+16 p_{x[k]} \times p_{y[k]}}\right] \frac{1}{n}
$$

143 If we define $p_{x}$ as $p_{x[k]}$ averaged across $k$ (and $p_{y}$ analogously), eq. [13] simplifies to

$$
P\left(S_{b}\right)=\frac{9 P\left(S_{a}\right)-3 p_{x}+4 p_{x} \times p_{y}-3 p_{y}}{9-12 p_{x}-12 p_{y}+16 p_{x} \times p_{y}}
$$

144 Eq. [13] or [14] permits us to calculate original sequence similarity, $P\left(S_{b}\right)$, given the raw sequence

145 similarity, $P\left(S_{a[k]}\right)$, and error rates $p_{x[k]}$ and $p_{y[k]}\left[\right.$ or $P\left(S_{a}\right), p_{x}$ and $\left.p_{y}\right]$. These equations

146 represent our method for correcting sequence similarity for sequencing error.

147 Simulation of reads. We applied our method for correcting sequence similarity to sets of 148 simulated reads. Reads with $n=300$ positions each were generated. As above, errors were 149 introduced under the modest assumptions that they 1) occur independently and 2) are in the form 150 of substitutions. Other conditions of the simulation are specified in Fig. 6.

151 Acknowledgements

152 We thank J. Tao and S. Hackmann (University of Florida) for reviewing the manuscript. This 153 work is supported by Agriculture and Food Research Initiative (AFRI) Competitive Grant no. 154 2017-67030-26589/project accession no. 1012177, Hatch Project accession no. 1002754, and 155 Hatch Project accession no. 1002352 from the USDA National Institute of Food and Agriculture.

\section{References}

1571 Bokulich, N. A. et al. Quality-filtering vastly improves diversity estimates from Illumina 158 amplicon sequencing. Nat Methods 10, 57-59, doi:10.1038/nmeth.2276 (2013). 
1592 Edgar, R. C. UPARSE: highly accurate OTU sequences from microbial amplicon reads.

$160 \quad$ Nat Methods 10, 996-998, doi:10.1038/nmeth.2604 (2013).

1613 Schloss, P. D., Gevers, D. \& Westcott, S. L. Reducing the effects of PCR amplification

162 and sequencing artifacts on $16 \mathrm{~S}$ rRNA-based studies. PLoS One 6, e27310,

163 doi:10.1371/journal.pone.0027310 (2011).

1644 Kozich, J. J., Westcott, S. L., Baxter, N. T., Highlander, S. K. \& Schloss, P. D.

165 Development of a dual-index sequencing strategy and curation pipeline for analyzing 166 amplicon sequence data on the MiSeq Illumina sequencing platform. Appl Environ 167 Microbiol 79, 5112-5120, doi:10.1128/AEM.01043-13 (2013).

1685 Edgar, R. C. \& Flyvbjerg, H. Error filtering, pair assembly and error correction for next169 generation sequencing reads. Bioinformatics 31, 3476-3482, 170 doi:10.1093/bioinformatics/btv401 (2015).

1716 Callahan, B. J. et al. DADA2: High-resolution sample inference from Illumina amplicon 172 data. Nat Methods 13, 581-583, doi:10.1038/nmeth.3869 (2016).

1737 Faith, D. P. Conservation evaluation and phylogenetic diversity. Biological conservation $174 \quad 61,1-10(1992)$.

1758 Lozupone, C. \& Knight, R. UniFrac: a new phylogenetic method for comparing microbial 176 communities. Appl Environ Microbiol 71, 8228-8235, doi:10.1128/AEM.71.12.82288235.2005 (2005). 
Table 1. Definition of terms in eq. [7] and [8] (and related terms)

\begin{tabular}{|c|c|c|c|c|c|}
\hline \multirow[b]{2}{*}{ Term $^{1,2}$} & \multirow[b]{2}{*}{ Expression } & Prior condition & \multicolumn{3}{|c|}{ Posterior condition } \\
\hline & & Similarity & Similarity & $\begin{array}{c}\text { Change (error) in } \\
X_{[k]}\end{array}$ & $\begin{array}{c}\text { Change (error) in } \\
Y_{[k]}\end{array}$ \\
\hline$P(\alpha)_{[k]}$ & $\left(1-p_{x[k]}\right)\left(1-p_{y[k]}\right)$ & $X_{[k]}$ and $Y_{[k]}$ similar & $X_{[k]}$ and $Y_{[k]}$ similar & - & - \\
\hline$P(\beta)_{[k]}$ & 0 & & & + & - \\
\hline$P(\gamma)_{[k]}$ & 0 & & & - & + \\
\hline$P(\delta)_{[k]}$ & $\frac{1}{3} p_{x[k]} \times p_{y[k]}$ & & & + & + \\
\hline$P(\varepsilon)_{[k]}$ & 0 & & $X_{[k]}$ and $Y_{[k]}$ disimilar & - & - \\
\hline$P(\zeta)_{[k]}$ & $p_{x[k]}\left(1-p_{y[k]}\right)$ & & & + & - \\
\hline$P(\eta)_{[k]}$ & $\left(1-p_{x[k]}\right) p_{y[k]}$ & & & - & + \\
\hline$P(\theta)_{[k]}$ & $\frac{2}{3} p_{x[k]} \times p_{y[k]}$ & & & + & + \\
\hline$P(A)_{[k]}$ & 0 & $X_{[k]}$ and $Y_{[k]}$ disimilar & $X_{[k]}$ and $Y_{[k]}$ similar & - & - \\
\hline$P(\beta)_{[k]}$ & $\frac{1}{3} p_{x[k]}\left(1-p_{y[k]}\right)$ & & & + & - \\
\hline$P(\Gamma)_{[k]}$ & $\frac{1}{3}\left(1-p_{x[k]}\right) p_{y[k]}$ & & & - & + \\
\hline$P(\Delta)_{[k]}$ & $\frac{2}{9} p_{x[k]} \times p_{y[k]}$ & & & + & + \\
\hline$P(E)_{[k]}$ & $\left(1-p_{x[k]}\right)\left(1-p_{y[k]}\right)$ & & $X_{[k]}$ and $Y_{[k]}$ disimilar & - & - \\
\hline$P(Z)_{[k]}$ & $\frac{2}{3} p_{x[k]}\left(1-p_{y[k]}\right)$ & & & + & - \\
\hline$P(H)_{[k]}$ & $\frac{2}{3}\left(1-p_{x[k]}\right) p_{y[k]}$ & & & - & + \\
\hline$P(\Theta)_{[k]}$ & $\frac{7}{9} p_{x[k]} \times p_{y[k]}$ & & & + & + \\
\hline
\end{tabular}

${ }^{1}$ As expected, $P(\alpha)_{[k]}+P(\beta)_{[k]}+P(\gamma)_{[k]}+P(\delta)_{[k]}+P(\varepsilon)_{[k]}+P(\zeta)_{[k]}+P(\eta)_{[k]}+P(\theta)_{[k]}=1$

$181 \quad{ }^{2}$ As expected, $P(A)_{[k]}+P(A)_{[k]}+P(\Gamma)_{[k]}+P(\Delta)_{[k]}+P(E)_{[k]}+P(Z)_{[k]}+P(H)_{[k]}+P(\Theta)_{[k]}=1$ 


\section{Figure legends}

184 Fig. 1. Cluster-based methods for handling sequence errors in ribosomal DNA sequences, illustrating their pitfalls. (A) Clustering thresholds are calibrated with mock communities of microbes to separate different sequences (species). Clusters are generally accurate (except for species 4 and 5 in this example). (B) Clustering cannot be calibrated with real communities because species are not known. Calibration from mock communities can be applied but may not be accurate (there are many clusters containing multiple species in this example). Sequences belonging to the same microbial species have the same color and number.

Fig. 2. Our method for handling sequencing error in ribosomal DNA sequences; this method focuses on correcting sequence similarity. Sequences become more dissimilar after introducing 194 sequencing errors, but similarities are corrected for error by application of eq. [13] or [14]. Dissimilarity is indicated by length of arrows. For simplicity, only a few arrows (those pertaining to the central sequence) are shown. Sequences belonging to the same microbial species have the 197 same color and number.

Fig. 3. Two DNA sequences before and after sequencing, illustrating of terms in our equations $X_{b}$ ) refer to conditions before and after sequencing. Each letter has a position $k$, and there are a 
207 Fig. 4. A given sequence position $(k)$ before and after sequencing, illustrating of terms in our 208 equations for correcting sequence similarity. (A) Condition where letters $X_{a[k]}$ and $Y_{a[k]}$ are

209 similar $\left[P\left(S_{a[k]}\right)=1\right]$. (B) Condition where letters $X_{a[k]}$ and $Y_{a[k]}$ are dissimilar $\left[P\left(D_{a[k]}\right)=1\right]$

210 In $(\mathrm{A}), P\left(S_{a 2[k]}\right)$ and $P\left(D_{b[k]}\right)$ are negative when $p_{x[k]}$ or $p_{y[k]}$ are positive, but they are depicted

211 as positive for illustration. In $(\mathrm{B}), P\left(D_{a 2[k]}\right)$ and $P\left(S_{b[k]}\right)$ are negative when $p_{x[k]}$ or $p_{y[k]}$ is

212 positive, but it is are depicted as positive for illustration.

213

214 Fig. 5. Comparison of raw sequence similarity $\left[P\left(S_{a}\right)\right]$ and original similarity $\left[P\left(S_{b}\right)\right]$. Error rates

215 vary from $0 \%\left(p_{x}=p_{y}=0\right)$ to $1 \%\left(p_{x}=p_{y}=0.01\right)$. Original similarity calculated from raw

216 similarity using eq. [14].

218 Fig. 6. Our method for correcting sequencing similarity, as applied to 15,000 simulated sequence

219 reads. (A) Reads before introduction of errors. Original similarity of reads was $97 \%\left[P\left(S_{b}\right)=\right.$

220 0.97]. (B) Reads after introduction of errors at a rate of $1 \%\left(p_{x}=p_{y}=0.01\right)$. (C) Reads after

221 correcting similarity values in (B) with eq. [14]. Panels (D-F) are analogous, except error rate was

$2220.2 \%\left(p_{x}=p_{y}=0.002\right)$. 
Figure 1

\section{(A) Clustering with mock communities}

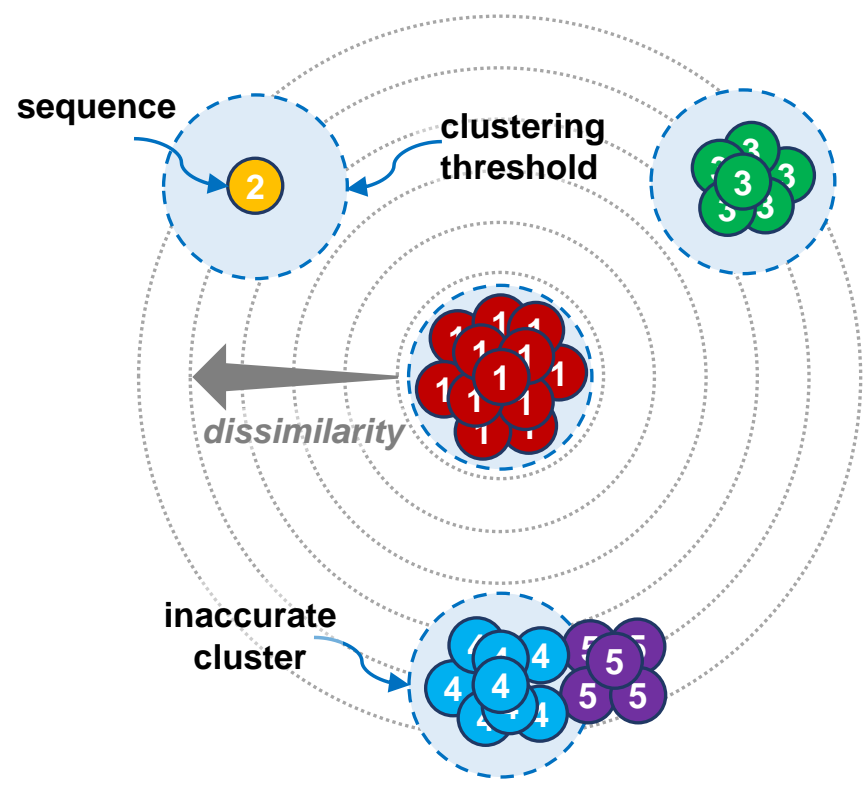

(B) Clustering with real communities

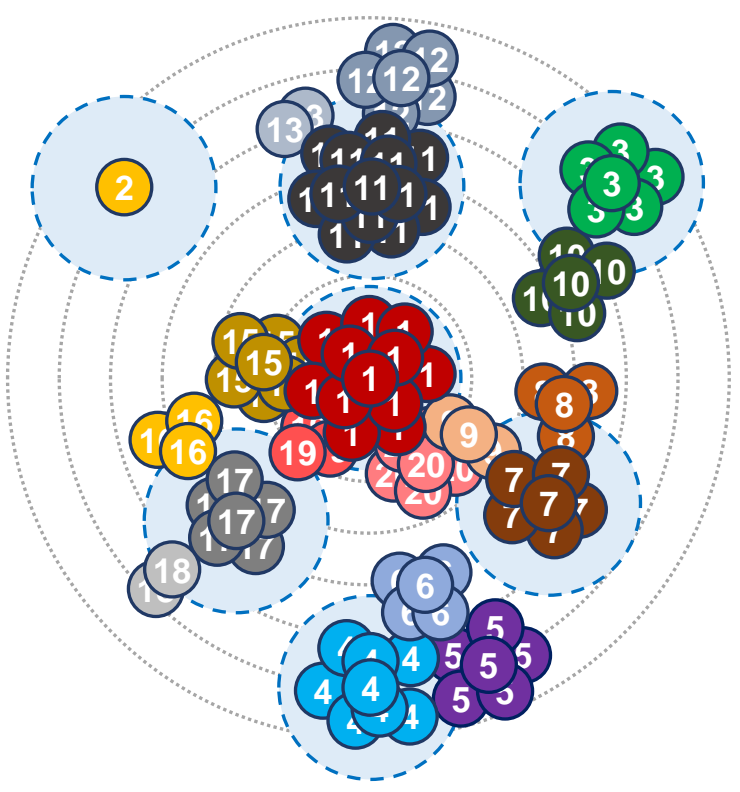


Figure 2

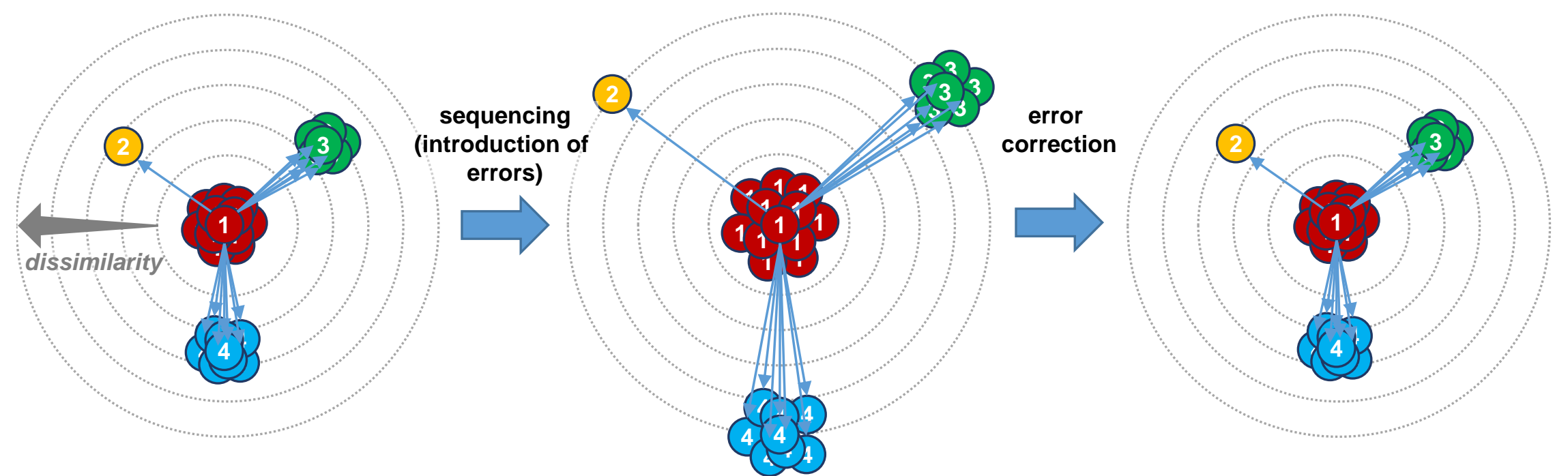




\section{Figure 3}

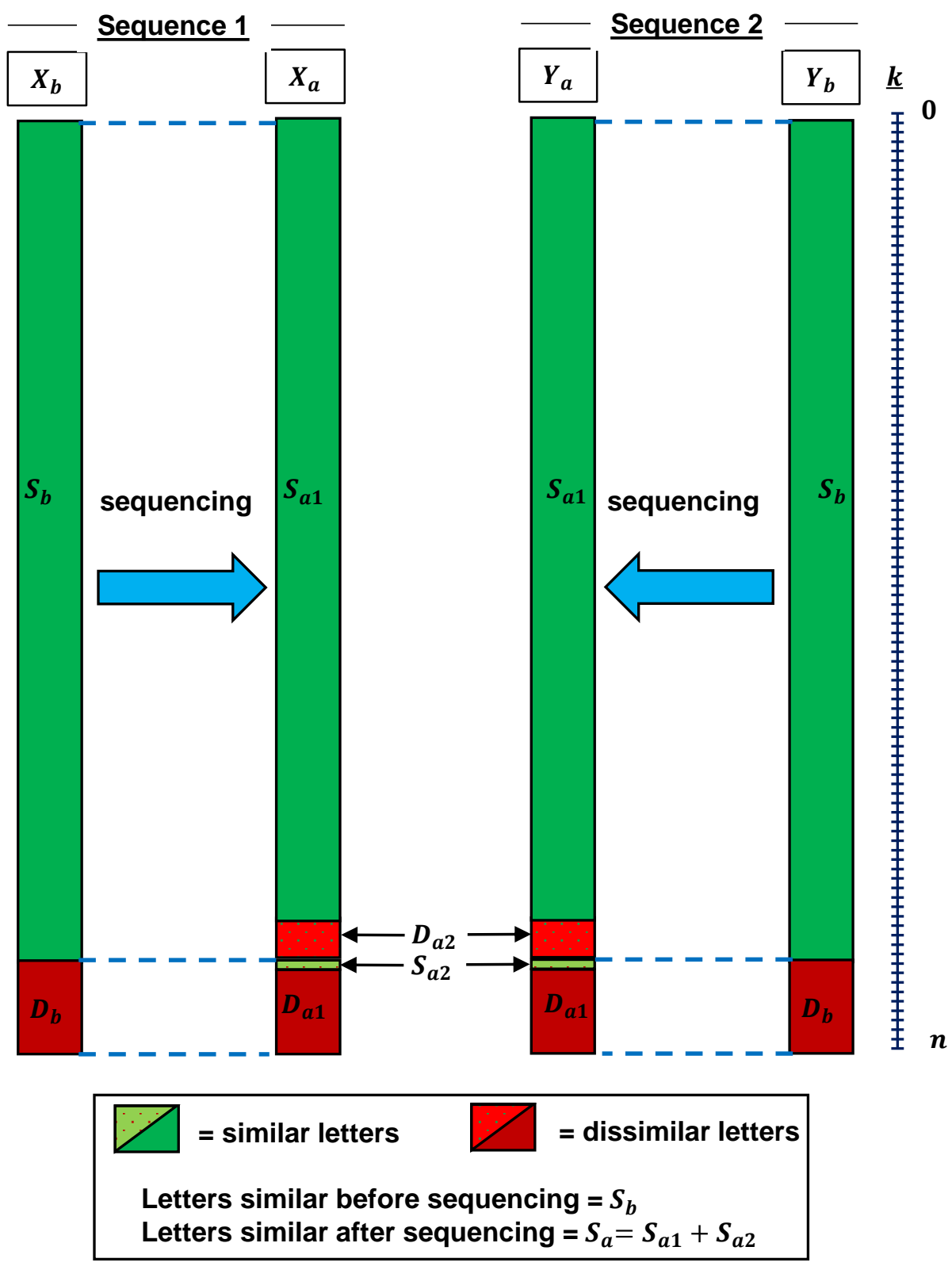




\section{Figure 4}

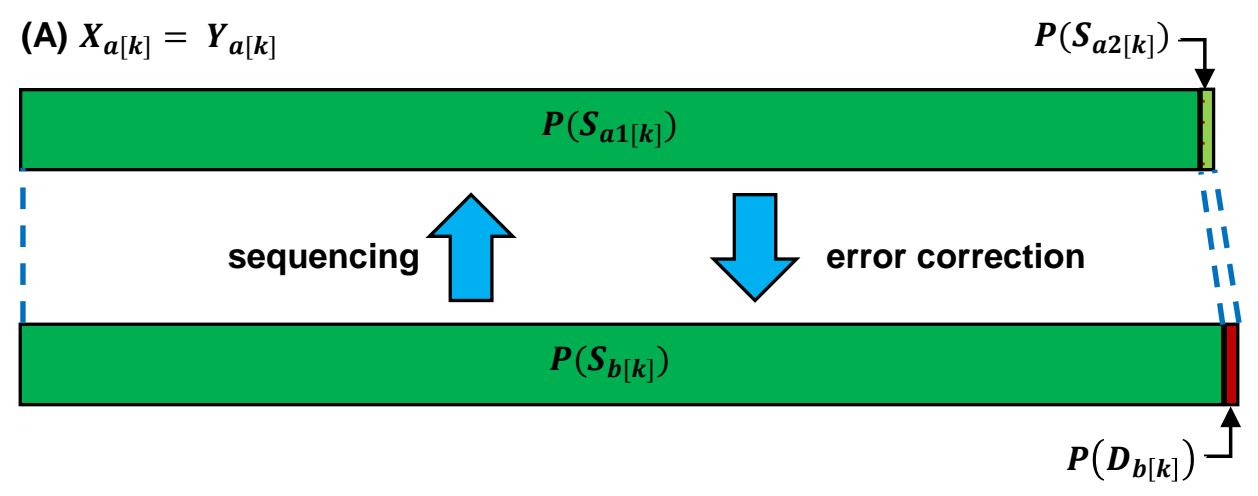

(B) $X_{a[k]} \neq Y_{a[k]}$

$$
P\left(D_{a 2[k]}\right)
$$

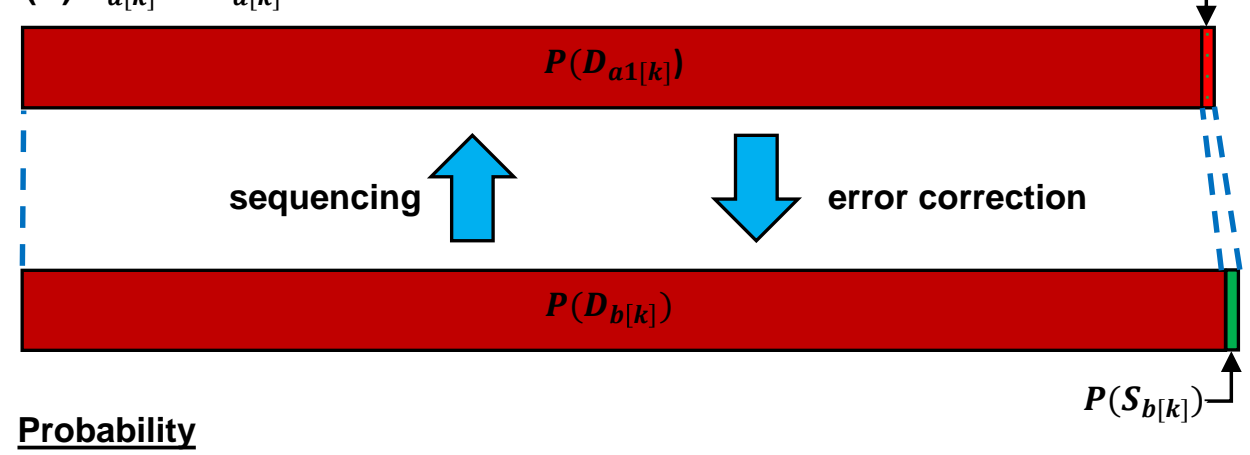

\section{0}

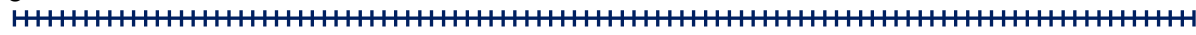

Raw similarity $=P\left(S_{a[k]}\right)=P\left(S_{a 1[k]}\right)+P\left(S_{a 2[k]}\right)$

Original similarity $=P\left(S_{b[k]}\right)$ 
Figure 5

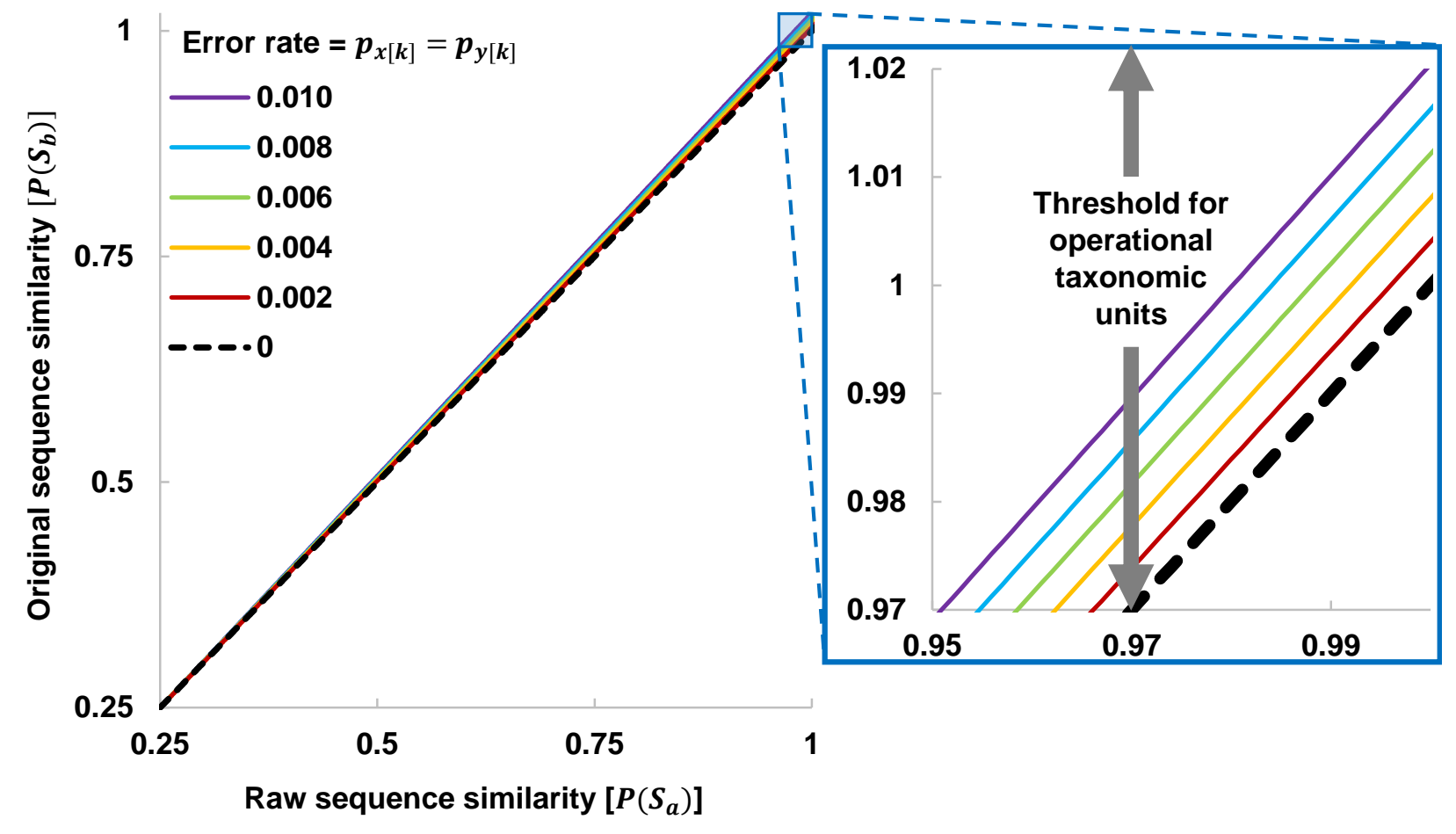


Figure 6
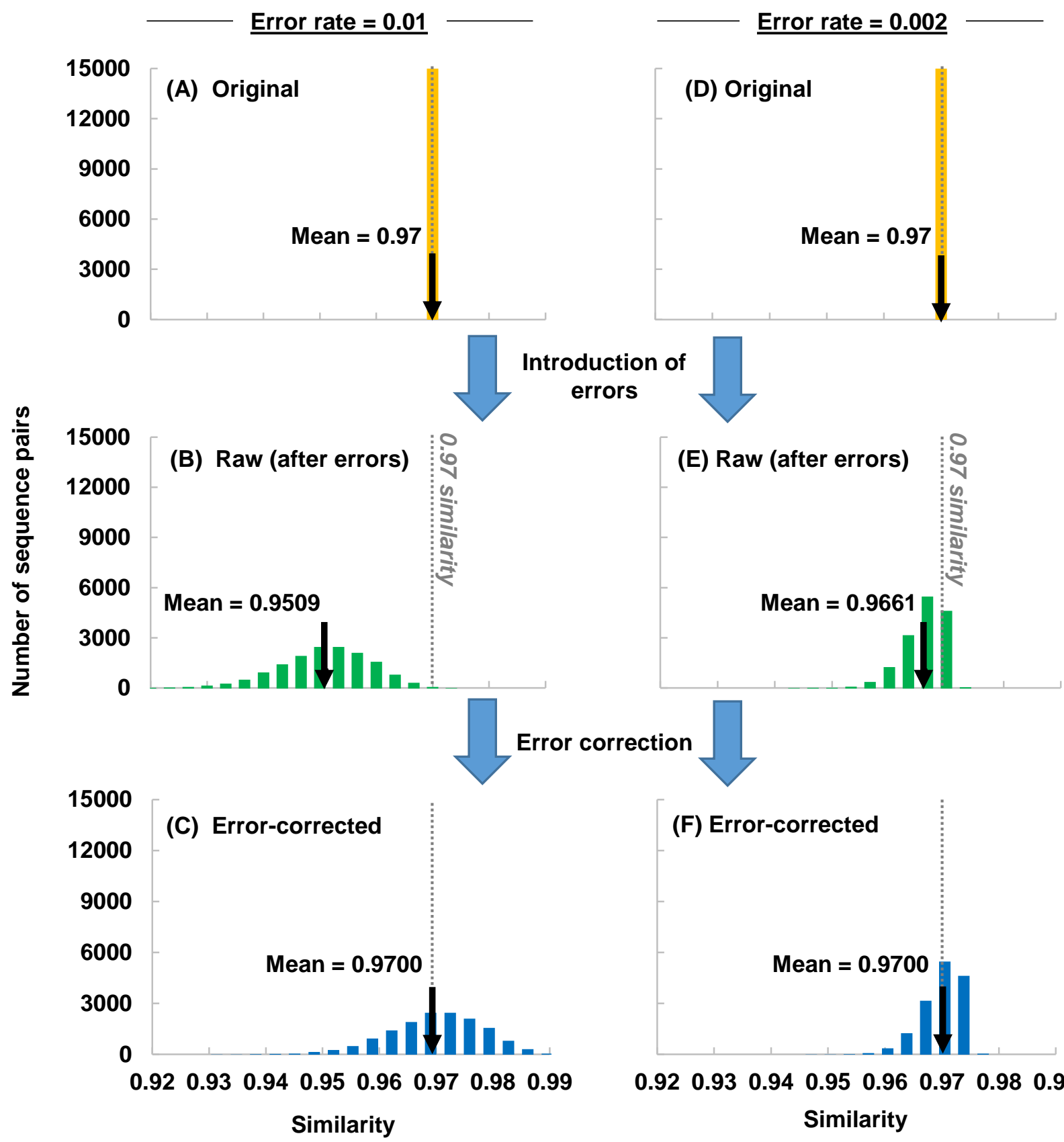